\title{
Classification biologique des lacs jurassiens à l'aide d'une nouvelle méthode d'analyse des peuplements benthiques ${ }^{1}$ III. Relations entre données biologiques et variables du milieu
}

\author{
J. Verneaux ${ }^{2}$ \\ A. Schmitt ${ }^{2}$ \\ V. Verneaux ${ }^{2}$
}

Mots clés : typologie des lacs, macrobenthos, facteurs écologiques, relations.

L'examen des relations entre les données biologiques (macrofaune benthique) et les variables du milieu, recueillies conjointement dans 9 lacs jurassiens, est effectué à l'aide d'une analyse de co-inertie. La forte corrélation entre les deux séries de variables valide la classification biologique proposée antérieurement et permet de préciser la signification des deux indices utilisés. L'indice biologique littoral (Bo) est associé au potentiel trophique du système et l'indice de déficit faunistique (Bz) traduit l'évolution de la qualité chimique de l'eau et des sédiments selon la profondeur.

Les lacs les plus biogènes, définis par les indices les plus élevés, sont corrélés positivement avec la minéralisation (conductivité, bicarbonates, $\mathrm{Ca} / \mathrm{Si}$ des sédiments) et avec l'oxygénation hypolimnétique (saturation en $\mathrm{O} 2$ dissous, potentiel redox) et correlés négativement d'une part, avec la teneur du sédiment en matière organique et les paramètres associés (COT, NT, Fe, Si) et d'autre part, avec la concentration en orthophosphates, en sels ammoniacaux et la biomasse algale (Chl a).

L'analyse de co-structure, en accord avec la tendance corrélative de $\mathrm{Bo}$ et $\mathrm{Bz}$, étaye une combinaison des deux critères dans un indice global de valeur biogène. Toutefois, la corrélation entre $B_{0}$ et $B_{Z}$ n'est pas généralisable et les deux indices sont utilisés pour définir le type biologique d'un lac et interpréter la note globale.

La discussion des résultats suggère une révision du classement des lacs par une recherche des conditions de transformation et de transfert de la matière assimilable aux organismes consommateurs.

Biological classification of nine French Jura lakes using a new comparative analysis of the macrobenthic communities III. Relationships between biological data and ecological factors

Keywords : lake typology, macrobenthos, ecological factors, relationships.

The relationships between biological data (macro-benthic fauna) and ecological descriptors (physical and chemical data), jointly collected in nine lakes of the French Jura, are studied using a co-inertia analysis. The high correlation between the two sets of variables confirms the validity of the biological classification described in two previous papers. The littoral biotic index (Bo) shows the trophic potential of the system and the taxonomic deficit index (Bz) accounts for the decrease of water and sediment quality according to the depth.

The most biogenic lakes, which are defined by the highest indices, are positively correlated with the mineralization (conductivity, bicarbonates) and the oxygen supply of the deep water (saturation rate, redox potential). On the other hand, the less biogenic lakes are positively correlated with the organic content of the sediment (COT, NT), other variables such as Fe or Si content and also with dissolved phosphorus $\left(\mathrm{PO}_{4}{ }^{3-}\right)$, ammonia $\left(\mathrm{NH}_{4}{ }^{+}\right)$and primary biomass $(\mathrm{Chl}$ a).

The results of the co-structure analysis, in agreement with the proximity of $\mathrm{Bo}$ and $\mathrm{Bz}$ in the factorial plane, leads to the combination of these two indices in a global biotic index. Whereas the co-variation of $\mathrm{Bo}$ and $\mathrm{Bz}$ cannot be considered as a general rule, the previous indices are useful to define the biological type of lakes.

From the discussion of the results, it is proposed that there should be a revision of the classification of lakes with a study about the environmental conditions connected with the transformation and the transfer of the assimilable matter to the consumers.

1. Recherches bénéficiant du soutien financier de l'Agence de l'Eau Rhône-Méditerranée-Corse.

2. Laboratoire de Biologie des Organismes et Ecosystèmes, Institut des Sciences et des Techniques de l'Environnement (I.S.T.E.) de l'Université de Franche-Comté, Place du Maréchal Leclerc, F:25030 Besançon cedex, France. 


\section{Introduction}

Dans deux précédentes publications Verneaux et al. (1993 a,b) ont exposé le protocole et les résultats d'une nouvelle classification biologique de neuf lacs jurassiens (Tableau 1) fondée sur l'analyse comparative de la macrofaune benthique d'après la variété générique, la densité et la nature de la macrofaune peuplant les sédiments fins.

La présente note, qui constitue le troisième volet du tryptique, est consacrée à l'analyse des relations entre le classement biologique proposé et les descripteurs physiques et chimiques du milieu mesurés conjointement (sauf pour les lacs Pavin et Aydat du Massif Central uniquement analysés biologiquement et intégrés à titre indicatif dans la figure 1).

Le traitement des données se voit assigner un triple objectif :

- donner la mesure des proximités entre les structures biologique et mésologique (analyse de co-inertie) afin de vérifier la validité du classement biologique proposé antérieurement (Verneaux et al. 1993);

- établir les corrélations interparamétriques entre variables biologiques et mésologiques;

- préciser la signification des indices $\mathrm{Bo}$ et $\mathrm{Bz}$ afin de permettre l'interprétation de la position de chaque lac dans le système proposé.

Enfin, les résultats obtenus sont discutés dans le cadre plus général de la biotypologie et des concepts relatifs au mode de fonctionnement des systèmes lacustres.

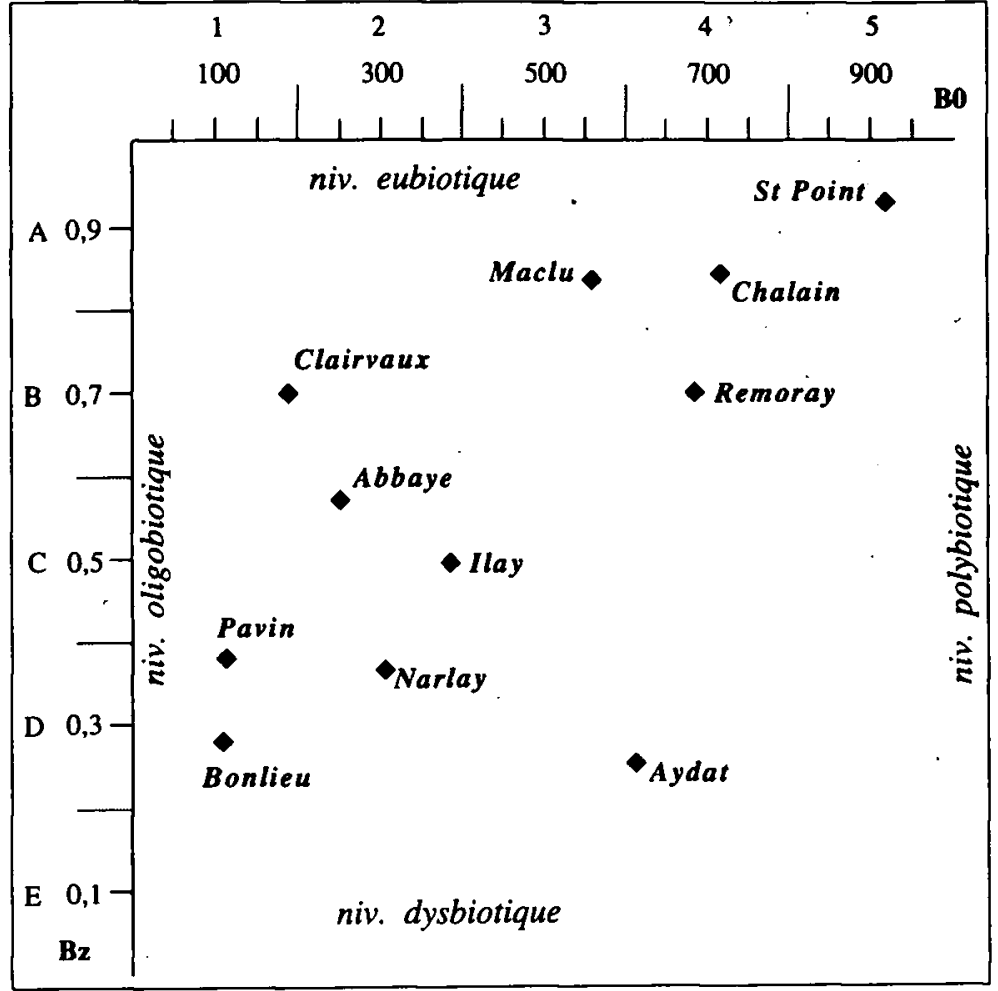

Fig. 1. Classement biologique des 11 läss d'après la macrofaune benthique. $\mathrm{Bo}=$ indice biologique littoral $; \mathrm{Bz}=$ indice de déficit faunistique.

Fig. 1. Biological classification of the eleven lakes according to the benthic macrofauna. $\mathrm{Bo}=$ littoral biological index $; \mathrm{Bz}=$ index of taxonomic deficit.

\section{Descripteurs utilisés et analyse des don- nées}

\subsection{Variables biologiques}

Le protocole d'analyse comparative, décrit antérieurement et appliqué à l'étude de 11 lacs, conduit à l'obtention des données présentées dans le tableau 2.

Tableau 1. Caractéristiques générales des 11 lacs étudiés (les lacs Pavin et Aydat, Massif Central, sont ajoutés uniquement dans le système de classifiçation biologique). Lacs : Abbaye, Bonlieu, Chalain, Clairvaux, llay, Maclu, Narlay, Remoray, St. Point, Pavin, Aydat.

Table 1. General characteristics of the eleven studied lakes (Pavin and Aydat lakes, located in the Massif Central, are added in the biological classification only). Lakes : Abbaye, Bonlieu, Chalain, Clairvaux, Ilay, Maclu, Narlay, Remoray, St. Point, Pavin, Aydat.

\begin{tabular}{|c|c|c|c|c|c|c|c|c|c|c|c|}
\hline & $A b$ & Bo & $C h$ & a & II & $\mathrm{Ma}$ & $\mathrm{Na}$ & $\mathrm{Pe}$ & Sp & $\mathrm{Pa}$ & $A y$ \\
\hline & 10 & 803 & 490 & 534 & 778 & 779 & 748 & 850 & 850 & 1197 & 825 \\
\hline & 80 & 17 & 232 & 56 & 72 & 2 & 41 & 95. & 398 & 44 & 60 \\
\hline$v\left(10^{6} \mathrm{~m}^{3}\right.$ & 5.8 & 1.5 & 44.0 & 5.3 & 7.7 & 2.6 & 8.2 & 9.6 & 81.0 & 23.0 & 4.2 \\
\hline$\overline{7}(\mathrm{r}$ & 7.2 & 8.8 & 19.0 & 9.5 & 10.7 & 11.8 & 20.0 & 10.1 & 20.3 & 54.9 & 7.4 \\
\hline & & & 32 & & 32 & 25 & 40 & 27 & 42 & 98 & \\
\hline
\end{tabular}


Tableau 2. Valeurs des descripteurs biologiques pour les 11 lacs étudiés.

vo : variété faunistique littorale (générique). do : densité de la faune en zone littorale (de 1 à $-2 \mathrm{~m}, \mathrm{ni} / 0.1 \mathrm{~m}^{2}$ ). qi : qualité de la faune aux profondeurs relatives successives (Verneaux et al. 1993b). dfi : coefficient de déficit faunistique aux profondeurs relatives successives (Verneaux et al. 1993a).

Table 2. Biological descriptors and values recorded in the eleven studied lakes.

vo : taxonomic variety (genera). do : total abundance of the benthic fauna in the littoral area $\left(-1\right.$ to $\left.-2 \mathrm{~m}, \mathrm{ni} / 0.1 \mathrm{~m}^{2}\right)$. qi : qualitative index of the benthic fauna at the different relative depths (Verneaux et al. 1993b). dfi ; index of taxonomic deficit at the different relative depths (Verneaux et al. 1993a).

\begin{tabular}{|c|c|c|c|c|c|c|c|c|c|c|c|}
\hline & $A b$ & Bo & Ch & $a$ & II & $\mathrm{Ma}$ & $\mathrm{Na}$ & $\mathrm{Pe}$ & Sp & $\mathrm{Pa}$ & Ay \\
\hline 10 & 26 & 18 & 44 & 27 & 33 & 32 & 33 & 44 & 54 & 12 & 28 \\
\hline do & 104 & 34 & 264 & 3 & 40 & 307 & 88 & 46 & 290 & 6 & 477 \\
\hline qo & 0,9 & 0,8 & 1,0 & 1,0 & 1,0 & 1,0 & 1,0 & 1,0 & 1,0 & 0,9 & 0,8 \\
\hline 1 & 0,8 & 0,4 & 1,0 & 1,0 & 0,8 & 0,9 & 0,7 & 0,9 & 0,9 & 0,9 & 0,5 \\
\hline 2 & 0,6 & 0,2 & 0,7 & 0,8 & 0,2 & 0,8 & 0,2 & 0,5 & 0,9 & 0,1 & 0,2 \\
\hline 3 & 0,4 & 0,1 & 0,4 & 0,3 & 0,1 & 0,5 & 0,1 & 0,2 & 0,7 & 0,1 & 0,1 \\
\hline$d f 1$ & 0,73 & 0,55 &, 00 & 00 &, 67 &, 94 &, 48 &, 66 & 0,97 & 0,54 & 0,45 \\
\hline$d f 2$ & 0,50 & 0,50 & 0,86 & 1,00 & 0,48 & 0,93 & 0,18 & 0,61 & 0,98 & 0,20 & 0,16 \\
\hline f 3 & 0,50 & 0,33 & 0,77 & 0,67 & 0,30 & 0,62 & 0,09 & 0,36 & 0,98 & 0,08 & 0,11 \\
\hline 30 & 265 & 105 & 715 & 196 & 390 & 560 & 310 & 690 & 920 & 111 & 612 \\
\hline $3 z$ & 0,58 & 0,29 & 0,85 & 0,71 & 0,50 & 0,84 & 0,37 & 0,70 & 0,92 & 0,38 & 0,25 \\
\hline
\end{tabular}

Les lacs se différencient dans un système de coordonnées rectangulaires portant en abscisses l'indice biologique littoral Bo et en ordonnées l'indice de déficit faunistique relatif $\mathrm{Bz}$. Ces deux descripteurs synthétiques ont été définis précédemment dans le premier volet du tryptique (Verneaux et al. 1993 a). Rappelons que Bo exprime le potentiel biogène de la zone littorale et que $\mathrm{Bz}$ traduit l'évolution des biocénoses benthiques (interface eau-sédiment) avec la profondeur.

$$
\begin{aligned}
& \text { Ont été calculés : } \\
& \text { Bo }: \text { vo } \sqrt{\text { do }} \\
& \text { B2 }=90(0.5 \sqrt{q 1 d f 1}+0.33 \sqrt{\mathrm{q} 2 \mathrm{df} 2}+0.17 \sqrt{\mathrm{q} 3 \mathrm{df} 3} \\
& \text { avec } \mathrm{dfi}=\mathrm{k} \text { vi/vo }
\end{aligned}
$$

La figure 1 rend compte du classement des lacs dans le système proposé. Le lac de St. Point (poly-eubiotique) s'oppose au lac de Bonlieu (oligo-dysbiotique) et le lac de Clairvaux (oligo-eubiotique) au lac d'Aydat (poly-dysbiotique).

\subsection{Variables mésologiques}

Relatifs à une colonne d'eau épilimnétique, à l'eau de contact à $3 / 4 \mathrm{Z}$ max (à $1 \mathrm{~m}$ du fond) et au sédiment à $3 / 4 \mathrm{Z}$ max, les 28 descripteurs sont portés dans le tableau 3 .

Le coefficient de saturation en oxygène dissous $(k$ sat) a été obtenu en effectuant le bilan des aires de dis- tribution (s) de 5 classes (k) de saturation dans un graphe espace/temps (hauteur d'eau comprise entre $Z$ $\max$ et $Z / 2$ et période située entre les deux circulations).

$$
\begin{aligned}
& \text { Ksat }=\Sigma \frac{\mathrm{Z} / 2}{\mathrm{Zmax}} \mathrm{sk} / 5 \mathrm{~S} \\
& \text { D'autre part } \mathrm{kN}=\log \mathrm{NO}_{3}^{-} / \mathrm{NH}_{4}^{+}
\end{aligned}
$$

\subsection{Traitement des données}

Les données ont été soumises à l'analyse en composantes principales (A.C.P. normée puisque les unités de mesure sont différentes) dans le cadre du logiciel A.D.E. (Chessel et al. 1995). Des analyses successives ont permis de traiter l'ensemble lacs-paramètres biologiques et l'ensemble lacs-paramètres physiques et chimiques (Fig. 3 et 4 ) et de définir les structures correspondantes. Un couplage des deux ensembles individus-variables a été réalisé dans une analyse de co-inertie (Dolédec et al. 1994). Cette analyse (Fig. 2), associée à un test dans lequel les deux ensembles sont combinés de manière aléatoire lors de 100 permutations, a permis d'évaluer le degré de concordance entre les structures précédemment définies.

\section{Résultats}

\subsection{Analyse de co-inertie}

Le tableau 4 met en évidence que les plans 1-2 de coinertie sont très sensiblement de même qualité que les 
Tableau 3. Descripteurs physiqués et chimiques de l'eau $(E ; C)$ et des sédiments $(S)$. Valeurs moyennes maximales et minimales.

$\mathrm{E}: \overline{\mathrm{x}}(\mathrm{IV}-\mathrm{XI}) 1$ mesure $/ 2 \mathrm{~m}, 2 /$ mois -3616 relevés. $\mathrm{C}: \overline{\mathrm{x}}$ (IV-XI) 1 mesure $/ 2 \mathrm{~m}, 2 /$ mois -288 relevés. $\mathrm{S}: \overline{\mathrm{x}}, \mathrm{n}$ éch $=\mathrm{f}(\mathrm{L})-2 \mathrm{séries} \mathrm{de} \mathrm{mesures} \mathrm{-}$ 116 relevés.

Table 3. Physical and chemical descriptors of water and sediments. Maximum and minimum average values.

$\mathrm{E}: \overline{\mathrm{x}}$ (IV-XI) 1sample/2 m, 2/months - 3616 data. C : $\overline{\mathrm{x}}$ (IV-XI) 1 sample/2 m, 2/months - 288 data. $S: \overline{\mathrm{x}}$, $\mathrm{n}$ samples $=\mathrm{f}(\mathrm{L})-2$ sampling periods -116 data.

\begin{tabular}{|c|c|c|c|c|c|}
\hline & & $E$ & C & & $S$ \\
\hline code & & $\begin{array}{l}\text { eau colonne } \\
\text { épilimn.(1-10m) }\end{array}$ & $\begin{array}{l}\text { eau contact (3/4 Zmax) } \\
\text { à } 1 \mathrm{~m} \text { du fond }\end{array}$ & & $\begin{array}{l}\text { sédiment } \\
3 / 4 \mathrm{Zmax}\end{array}$ \\
\hline E1 & $\mathrm{Zs}(\mathrm{m})$ & $2,7-5,7$ & & & \\
\hline$E_{2}$ & $C\left(\mu S . . \mathrm{Cm}^{-1}\right)$ & $186-283$ & & & \\
\hline E3. & Chla max $\left(\mu \mathrm{g} \cdot \ell^{-1}\right)$ & $3,3-27,4$ & & & \\
\hline E4 & Chla $\left(\mu \mathrm{g} \cdot \ell^{-1}\right)$ & $1,8-9,4$ & & & \\
\hline E5 & $\mathrm{pH}$ & $7,8-8,7$ & & & \\
\hline E6 & $\mathrm{HCO}_{3}^{-}\left(\mathrm{mg} \cdot \ell^{-1}\right)$ & $137-223$ & & & \\
\hline E7 & k sat. & $0,35-0,88$ & & code & \\
\hline E8 & $\mathrm{NO}_{3}-\left(\mathrm{mgl} \ell^{\ell-1}\right)$ & $0,050-1,625$ & $0,13-3,62$ & $\mathrm{C}_{1}$ & \\
\hline E9 & $\mathrm{NH}_{4}^{+}\left(m g l l^{\ell-1}\right)$ & $0,011-0,046$ & $0,10-1,46$ & $\mathrm{C} 2$ & \\
\hline E10 & $\mathrm{PO}_{4}{ }^{3-}\left(\mathrm{mg} \cdot \ell^{-1}\right)$ & $<0,004-0,046$ & $0,207-1,05$ & C3 & \\
\hline $\mathrm{C} 4$ & $\mathrm{~T}^{\circ}\left({ }^{\circ} \mathrm{C}\right)$ & & $6,80-9,80$ & & \\
\hline $\mathrm{C} 5$ & $\mathrm{kN}$ & & $-0,84-1,48$ & & \\
\hline $\mathrm{C} 6$ & $\mathrm{eH}(m V / A g C l)$ & & $45-191$ & & \\
\hline$C 7$ & $\mathrm{O}_{2}\left(\mathrm{mg} \cdot \ell^{-1}\right)$ & & $1,0-6,7$ & & \\
\hline cs & sat (\%) & & $<1-61$ & & \\
\hline $\mathrm{C9}$ & $\mathrm{Fe}$ (mg.l-1) & & $0,03-0,52$ & & \\
\hline$C_{10}$ & Si (mg.l-1) & & $1,12-2,26$ & & \\
\hline s1 & MO (\% MS) & & & & $6,10-28,90$ \\
\hline S2 & COT (\%MS) & & & & $3,10-14,82$ \\
\hline S3 & NT (\%MS) & & & & $0,20-1,25$ \\
\hline S4 & $\mathrm{CaCO}(\% \mathrm{MS})$ & & & & $29,8-84,20$ \\
\hline S5 & $\mathrm{SiO} 2(\% \mathrm{MS})$ & & & & $4,60-26,70$ \\
\hline S6 & $\mathrm{Ca} / \mathrm{Si}$ & & & & $0,91-15,10$ \\
\hline S7 & $\mathrm{Fe}(\% \mathrm{MS})$ & & & & $0,52-3,20$ \\
\hline S8 & $\mathrm{C} / \mathrm{N}$ & & & & $8,80-12,70$ \\
\hline
\end{tabular}

plans 1-2 de chaque analyse. De plus, il apparaît que les deux projections sont reliées par les corrélations entre systèmes de coordonnées de même rang : $\mathrm{r} 2=$ 0,9149 et 0,7531 (Fig. 2a).

Le test de permutation (ADE, Chessel et al. 1995) confirme ainsi le caractère non aléatoire de la liaison faune-milieu, les corrélations observées étant significatives au seuil de $1 \%$.

Cette très nette corrélation entre les ordinations biologique et mésologique est visualisée par la figure $2 \mathrm{~b}$, qui présente les projections du nuage des 9 lacs selon les deux ensembles de critères. 
Tableau 4. Inertie projetée sur les axes 1 et 2 (espaces initiaux et espace de co-inertie et corrélation. $\left(\mathrm{r}^{2}\right)$ entre les systèmes de coordonnées.

Table 4. Projected inertia on the first and second axis (starting spaces and co-inertia space) and $\mathrm{r}^{2}$ values (correlation between the two sets of coordinates resulting from the co-inertia analysis).

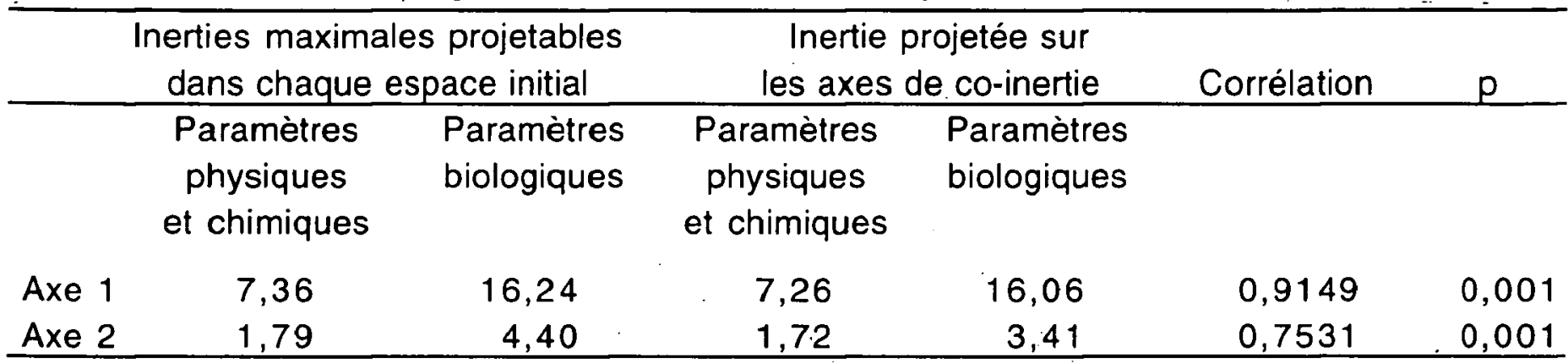

(a)

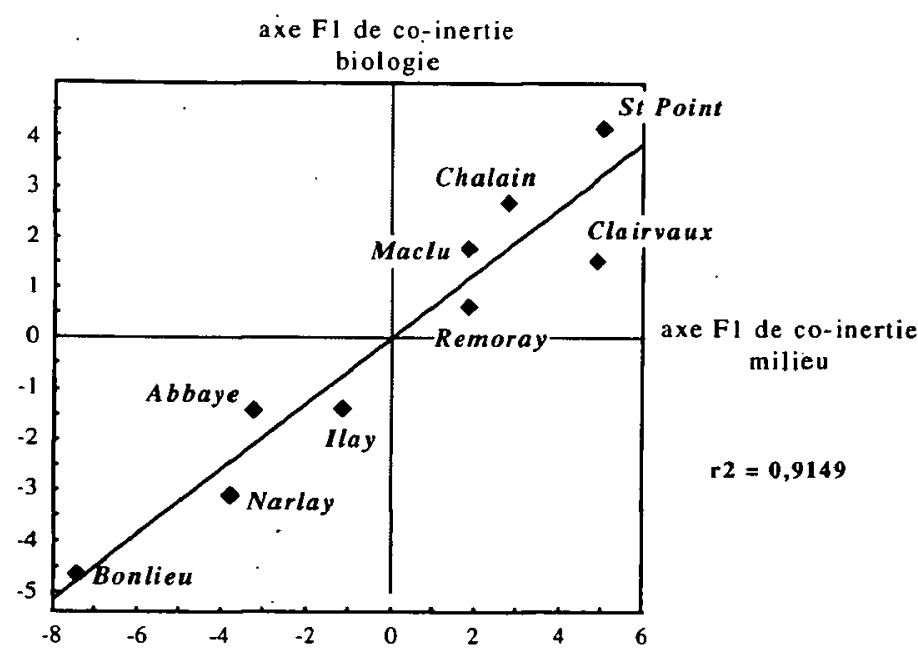

(b)

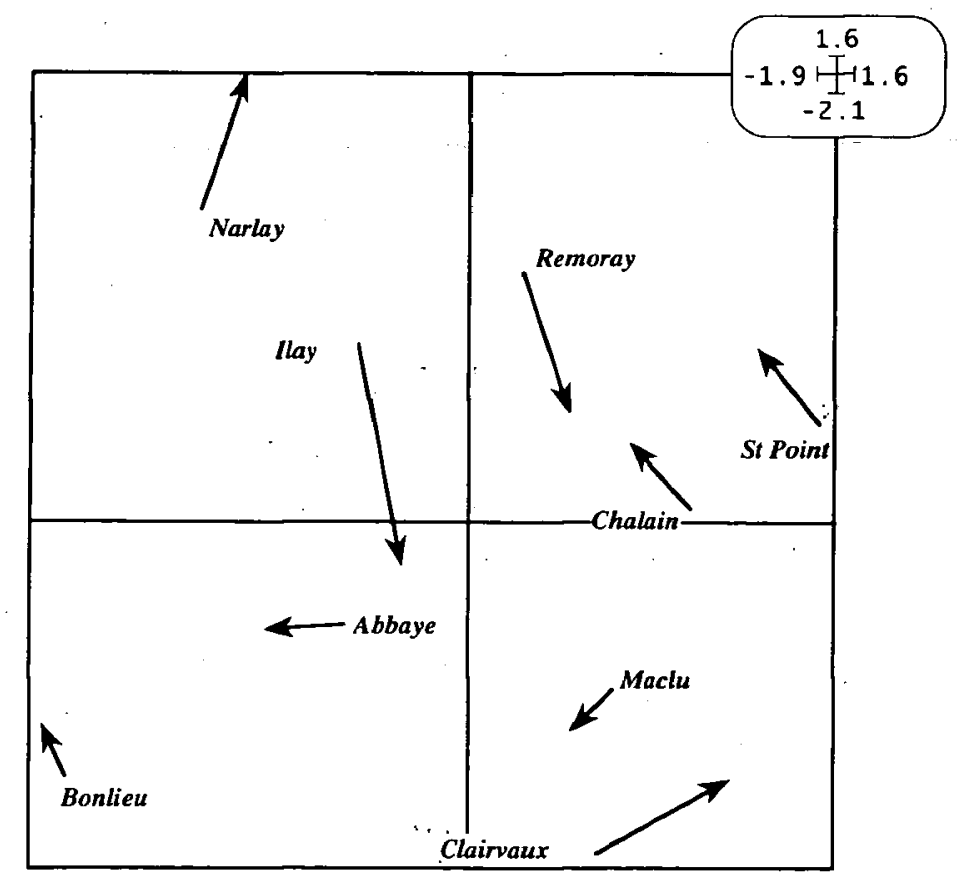

Fig. 2. Analyse de co-inertie. 2a : corrélation entre les deux classements selon les 2 axes $F 1$ de co-inertie. $2 b$ : position des lacs dans le plan des 2 premiers axes d'analyse (F1-F2). La base de la flèche indique la position définie par les variables biologiques et la pointe de la flèche indique celle définie par les paramètres du milieu.

Fig. 2. Co-inertia analysis. 2a : correlation between the environmental and the biological ordinations of lakes. $2 b$ : positions of lakes on the F1-F2 factorial plane. The bottom of the arrow indicates the position of lakes resulting from the biological ordination and the head links the position resulting from the environmental ordination.
Le classement des lacs, de St. Point à Bonlieu, est particulièrement évident sur la figure $2 \mathrm{a}$ (plan des deux premiers axes) ainsi que selon le premier axe de la figure $2 \mathrm{~b}$. Les écarts entre les deux types de classement, biologique et mésologique, sont toujours faibles sur le premier axe et plus marqués sur le second, surtout pour les situations intermédiaires (Ilay- Remoray-Narlay). Le premier axe paraît exprimer la différenciation primordiale des types et le second, l'impact de particularités structurales ou induites par des actions anthropiques (fosse isolée à une extrémité du lac d'Ilay, pollutions des lacs de Remoray et surtout de Narlay).

\subsection{Corrélations et carte factorielle}

Les relations entre descripteurs apparaissent surtout sur la figure 3 et les proximités entre les paramètres et les lacs s'observent dans le plan de la figure 4.

Le cercle des corrélations (Fig.3) met en évidence :

a) la présence de deux variables indépendantes et antagonistes : le pH épilimnétique associé à l'activité métabolique et le rapport $\mathrm{C} / \mathrm{N}$ des sédiments exprimant, par les valeurs s'écartant du seuil de 10, l'origine dominante et le degré de transformation de la matière organique (apports allochtones et périphériques ou matériel planctonique, Verneaux et al. 1991).

La situation des orthophosphates provient du fait que seul le lac de Narlay (Fig. 4) présente des concentrations notables de phosphates résiduels en zone épilimnétique ( $\left.\bar{x}=46 \mu \mathrm{g} . \mathrm{l}^{-1}\right)$,

b) quatre noyaux antagonistes de variables redondantes ou corrélées :

- le noyau a exprime le potentiel trophique des systèmes avec les sels de calcium et les nitrates. La présence dés nitrates dans ce groupe, et non à proximité des phosphates et de la chlorophylle, s'expliqué par le fait que les concentrations maximales $\left(\bar{X} \approx 1 \mathrm{mg} \cdot \mathrm{l}^{-1}\right)$ 


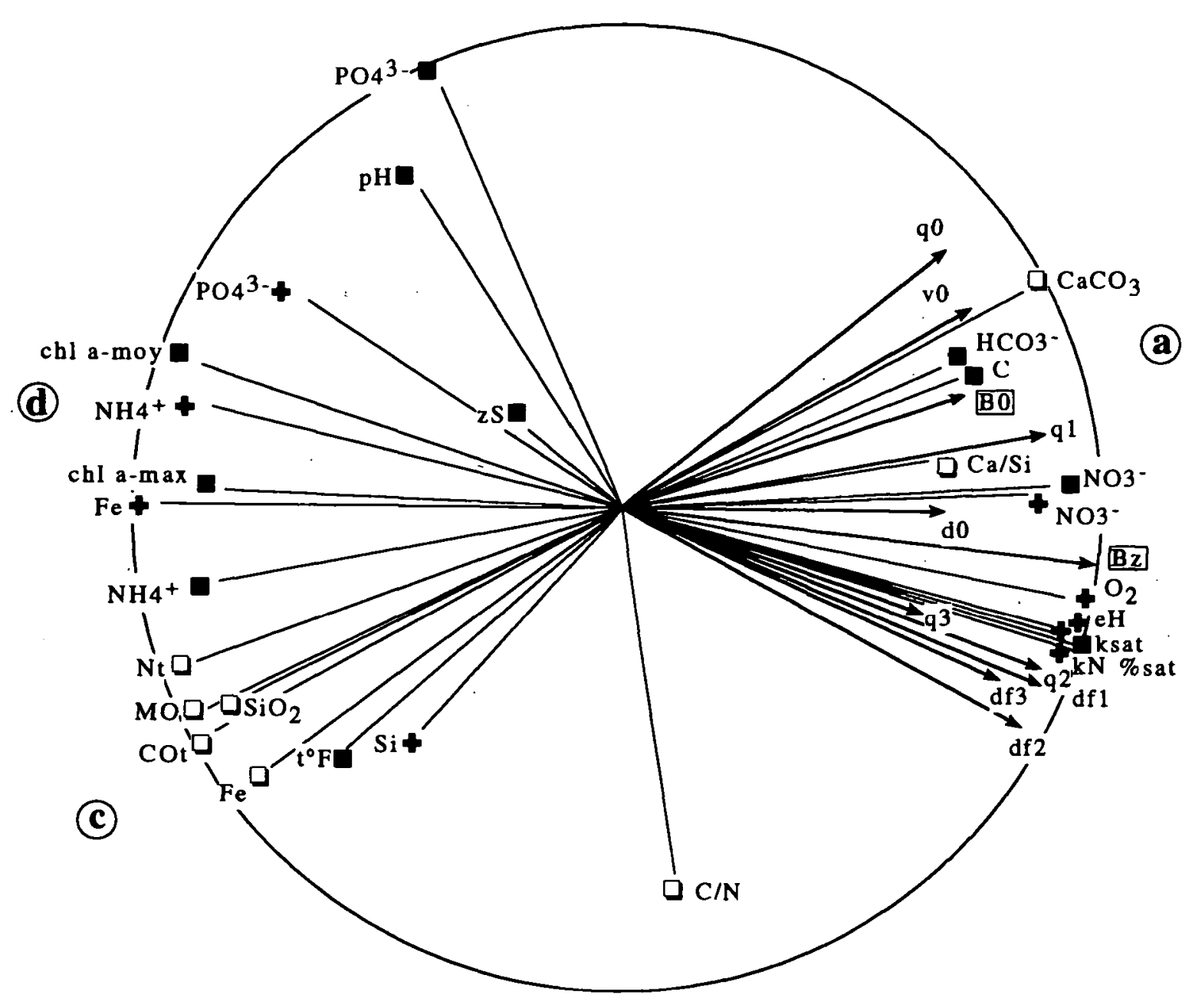

(b)

Fig. 3. Cercle des corrélations - ACP normée - 11 descripteurs biologiques et 28 paramètres physiques et chimiques. - 9 lacs. Symboles cf. Fig. 4 .

Fig. 3. Correlation circle - P.C. normalized analysis - eleven biological descriptors and twenty eight physical and chemical variables - nine lakes. Symbols as in Fig. 4.

relevées en zone euphotique sont associées aux lacs polybiotiques et n'apparaissent pas comme limitantes par excès (Fig. 4) ;

- le noyau c, anticorrélé au noyau a, traduit l'accumulation de matière organique résiduelle associée à la silice et au fer dans des complexes de néoformation (Verneaux et al. 1987-1991);

- le noyau b regroupe les descripteurs associés à 1'oxygénation des eaux profondes ( $3 / 4 \mathrm{Zmax})$ et au coefficient global d'oxygénation (k sat. , colonne) ;

- le noyau d, anticorrellé au noyau b, associe le fer, les sels ammoniacaux et les orthophosphates des eaux de contact à la biomasse algale (Chl a).

En dehors du peu de signification de la transparence, dont les variations dans les lacs de faibles ou moyennes dimensions sont liées à divers facteurs notamment la remise en suspension des sédiments par le vent (Martin 1994), le fait le plus remarquable consiste en l'antagonisme entre biomasse algale et conditions écologiques favorables au développement des peuplements benthiques consommateurs (noyaux a et $\mathbf{b}$ ).

On observe l'association des descripteurs biologiques littoraux, en particulier l'indice littoral Bo, aux conditions trophiques du groupe a et celle des descripteurs biologiques de la zone profonde, en particulier l'indice de déficit faunistique $\mathrm{Bz}$, aux conditions d'oxygénation (ou d'oxydo-réduction du groupe b).

Ces données permettent d'envisager une interprétation de la classification fonctionnelle des lacs fondée sur les conditions de transformation et de transfert de la matière (allochtone ou autochtone) disponible aux organismes consommateurs.

La carte factorielle montre que les lacs les moins biogènes relativement à la macrofaune benthique (lacs dysbiotiques, Fig. 1), sont ceux qui sont les plus désoxygénés et qui présentent soit les sédiments les plus riches en matière organique (lacs polyhumiques l'Abbaye, Ilay, COT $>10 \%$ m.s.) soit les plus fortes 


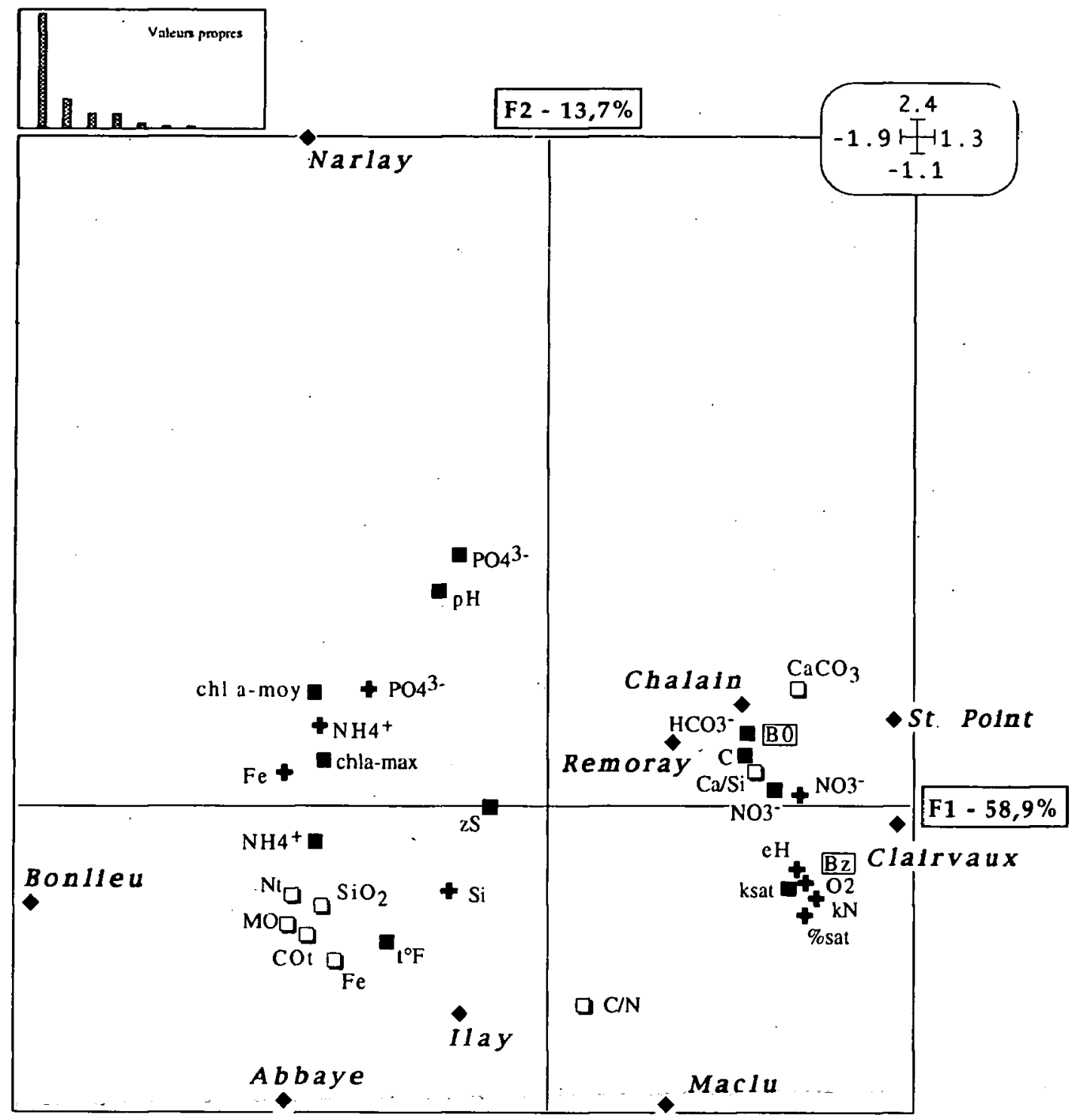

Fig. 4. Positions des 9 lacs et des descripteurs dans le plan des deux premiers facteurs d'une ACP normée.

: paramètres de l'eau colonne centrale. + : paramètres de l'eau de contact. $\square$ : paramètres des sédiments. $\square$ : descripteurs biologiques.

Fig. 4. Ordinations of nine lakes and environmental descriptors in the F1-F2 factorial plane of a P.C. normalized analysis.

- : variables in surface water (column). + : variables in deep water. $\square$ : sediment variables. $\square$ : biological descriptors.

concentrations en orthophosphates et en pigments photosynthétiques (lacs polytrophes-Narlay, Chl a. $\overline{\max }$ $13.6 \mu \mathrm{g} .1-1)$, soit les deux comme à Bonlieu (Chl a. $\overline{\max }=27,4 \mu \mathrm{g} .1-1, \mathrm{COT}=14 \% \mathrm{~m} . \mathrm{s}$.). Dans ces deux derniers lacs les concentrations en pigments totaux sont nettement plus élevées, par suite d'une «fleur d'eau» à Oscillatoria rubescens.

Les rôles majeurs de l'oxygène dissous et de la teneur en matière organique des sédiments dans la différenciation biologique des lacs ont déjà été signalés par plusieurs auteurs (Lundbeck 1926, Brundin 1949, Prat 1978, Petersen et al. 1986, Lafont et al. 1991, Mouthon 1992). Brundin (1949) soulignait également l'absence de relation constante entre les conditions d'oxydo-réduction et le «niveau trophique» d'un système lacustre.
Ainsi, le lac de St. Point, à fort potentiel trophique exprimé par un indice biologique littoral (Bo) élevé, n'est pas désoxygéné en profondeur et accumule relativement peu de matière organique résiduelle $(\mathrm{MO}<$ $10 \% \mathrm{~ms}$ ).

L'axe F1 nettement dominant, portant près de $60 \%$ de l'inertie, exprime conjointement le gradient dı qualité physico-chimique et l'aptitude biogène résultante du système (synthèse de Bo et de $\mathrm{Bz}$ ).

L'axe F2 différencie surtout la nature et l'origine des apports : les lacs de l'Abbaye et du Maclu sont péritrophes dominants alors que le lac de Narlay est pélagotrophe dominant (forte biomasse algale induite par des apports de nutriments - Verneaux et al. 1991). 
Cette interprétation est confirmée par la position du rapport $\mathrm{C} / \mathrm{N}$ dont les valeurs élevées $(>12)$ traduisent une transformation incomplète d'éléments à fort $\mathrm{C} / \mathrm{N}$ issus d'apports terrigènes ou des ceintures végétales (lacs de l'Abbaye et de Bonlieu).

Le lac de St. Point, poly-eubiotique (Fig. 1), est désigné comme lac de référence et le lac de Bonlieu, oligo-dysbiotique (Fig. 1) passant à la tourbière, se révèle le lac le moins biogène de l'échantillon analysé ; il est polyhumique (environnement forestier) et durablement hypoxique.

\section{Relation entre Bo et $\mathrm{Bz}$}

En accord avec l'analyse de co-structure (Fig. 2a), la proximité des indices $\mathrm{Bo}$ et $\mathrm{Bz}$ dans les graphes des figures 3 et 4 confirme l'existence d'une relation entre ces deux descripteurs, suggérée par la tendance à une organisation de la plupart des lacs étudiés selon la diagonale de la figure 1 .

Conformément à la dynamique du complexe mésologique lacustre, les deux variables ne sont pas indépendantes, bien que dans certains cas (Clairvaux et Aydat, Fig. 1) leurs spécificités indicatives apparaissent.

Dans le cas du lac de Clairvaux, aux sédiments pauvres en matière organique (COT $<3 \% \mathrm{~m}$.S.) et peu désoxygéné, sauf en Z3, la production littorale et épilimnétique est inhibée par une très forte précipitation calcique et les turbidités élevées dues à l'action des vents et aux précipitations (aspect laiteux, Verneaux et al. 1993 a). Dans le cas du lac d'Aydat ce sont les excès de nutriments allochtones qui génèrent de fortes biomasses algales et des excédents de matière organique, réductrice, en zone profonde (Alaoui \& Mahmdi, 1985, et mesures effectuées en 1993 au cours de la campagne d'échantillonnage du macrobenthos).

Par ailleurs, si un lac oligotrophe est nécessairement oligobiotique, il peut selon les cas se révéler eubiotique (Bz proche de 1) ou dysbiotique (Bz faible, cf lac Pavin, Fig. 1).

Ces observations relatives à la concordance et à la discordance des deux indices conduisent à envisager une méthode comprenant un indice biologique résultant, issu de la combinaison des deux indices, complété par les indications propres à chaque indice pour mieux définir le type biologique des lacs.

\section{Conclusions et discussion}

La confrontation des données biologiques et mésologiques à l'aide d'une analyse de co-inertie met en évidence la très forte corrélation entre les deux séries de descripteurs, valide la classification biologique propo- sée antérieurement et permet une interprétation fonctionnelle de la combinaison des deux indices utilisés.

Le potentiel trophique des lacs influence l'indice biologique littoral Bo corrélé à la minéralisation exprimée globalement par la conductivité et les paramètres associés (bicarbonates, calcium, rapport $\mathrm{Ca} / \mathrm{Si}$ des sédiments). Les concentrations moyennes en nitrates épilimnétiques, lorsqu'elles sont inférieures à un seuil de l'ordre du $\mathrm{mg} \mathrm{l}^{-1}$ dans les conditions indiquées, figurent dans ce groupe paramétrique. Pour les orthophosphates, à l'exception du lac de Narlay, pollué par des émergences karstiques profondes, toutes les concentrations moyennes maximales sont inférieures à $15 \mu \mathrm{g} \mathrm{l}^{-1} \mathrm{et}$ des valeurs inférieures à $4 \mu \mathrm{g} \mathrm{l}^{-1}$ sont fréquemment relevées.

On note que les valeurs limitantes approchées mentionnées par Rast (in Ryding \& Rast 1994) sont de $5 \mu \mathrm{g} \mathrm{l}^{-1}$ pour le phosphore et de $20 \mu \mathrm{g} \mathrm{l}^{-1}$ pour l'azote biodisponibles et les concentrations supérieures relevées en zone euphotique en fin de stratification estivale peuvent être considérées comme excédentaires (teneurs résiduelles non transférées).

Ces résultats confirment ceux d'auteurs comme Hutchinson (1957), Hayes \& Philipps (1958), Rigler (1964), Johnson \& Wiederholm (1989) qui mettent en doute la validité des concentrations, en nutriments ou en pigments photosynthétiques, mesurées dans la masse d'eau, en tant qu'indicateurs de statuts trophiques. Au-delà de certains seuils, pouvant être très faibles dans le cas de transfert optimal, les concentrations ainsi mesurées constitueraient plutôt des indications de dysfonctionnement.

Le fait que la chlorophylle a soit maximale dans les lacs les moins biogènes (Fig. 4) ne signifie pas que la production primaire de ces lacs soit supérieure à celle des lacs polybiotiques, mais que les conditions chimiques associées ne permettent pas le développement des organismes consommateurs ainsi que le transfert de la biomasse produite. L'analyse montre que le caractère dysbiotique des lacs peut résulter aussi bien d'un excès de nutriments (Narlay, Aydat) que de l'accumulation de matériel périphérique inicomplètement transformé (Abbaye, Ilay, Maclu) et que les deux causes peuvent être conjointes (Bonlieu).

Si l'on considère que la réaction biologique interne que constitue la prolifération algale est généralement engendrée par des apports nutritionnels externes, nos observations sont en accord avec la proposition de Wetzel (1975) qui associe les formes de dystrophie à l'allotrophie et l'eutrophie à l'autotrophie. Toutefois, on peut se demander si, dans un système ouvert, cette différenciation ne présente pas un caractère artificiel. 
Par exemple en un instant $t+x$ les nutriments apportés au lac de St. Point par le Doubs (allotrophie) engendrent de fortes proliférations algales intégrant une dynamique autotrophe.

Comme dans les analyses effectuées par Petersen et al. (1986) ou Johnson \& Wiederholm (1989), l'indice de déficit faunistique $(\mathrm{Bz})$ est corrélé positivement à l'oxygénation et négativement à la teneur des sédiments en matière organique résiduelle. Le seuil, repéré par les valeurs relevées dans les lacs eubiotiques . (Clairvaux, Chalain, St. Point - Fig. 1) se situe aux environs de $10 \%$ m.s. Dans le même ordre d'idée; Håkanson (1983) limite la série oligotrophie-eutrophie aux teneurs en MO inférieures à $20 \%$ m.s. Au-delà de cette valeur, les lacs polyhumiques entrent dans la catégorie des systèmes dystrophes. Toutefois, les descripteurs $\mathrm{O}_{2}$ et $\mathrm{MO}$ constituent un couple de variables interactives et il est possible que les teneurs favorables de $\mathrm{MO}$ augmentent avec la disponibilité en oxygène dissous (vecteurs lotiques, morphométrie, renouvellement de la masse d'eau ...) et que les teneurs inférieures à $10 \%$ m.s. soient limitantes par défaut (par exemple à Clairvaux).

L'indice Bz traduit la qualité du complexe eau/sédiment exprimant elle-même l'aptitude du système à transformer la matière disponible (minéralisation) et à la transférer aux organismes consommateurs en limitant le flux de MO sédimentée (Martin 1994).

Les lacs polyhumiques sont à la fois oligobiotiques et dysbiotiques (Bonlieu, l'Abbaye, Ilay) mais peuvent , comme à Bonlieu, générer des biomasses algales résiduelles importantès $\left(\sum\right.$ pigments $\max =86 \mu \mathrm{g} / 1^{-1}$ à Bonlieu).

L'assimilation quasi-officielle du phénomène d'eutrophisation à la pollution (O.C.D.E. 1982 in Ryding \& Rast 1994) ne contient pas seulement une impropriété terminologique, due à une déformation de la proposition du pédologue Weber (1907) ce qui serait de peu d'importance, mais introduit une confusion au plan conceptuel, entre une augmentation d'intensité métabolique et des formes de dysfonctionnement.

Cependant, dès 1934 Grote opposait logiquement l'oligotrophie à la polytrophie et la dystrophie à l'eutrophie caractérisant les «harmonische See Typen» qui seraient, selon nos résultats, caractérisés par de faibles concentrations de matière résiduelle, tant dissoute que sédimentée, quel que soit leur degré trophique ou l'intensité de leur métabolisme.

L'analyse des relations entre le classement biologique, en tant qu'expression synthétique résultante, et les composantes mésologiques montre qu'il convient de distinguer le niveau trophique (aspect quantitatif) et le statut trophique (aspect qualitatif) exprimant respectivement le potentiel nutritif et le mode de fonctionnement des systèmes lacustres (devenir du potentiel). De ce point de vue, un statut d'eutrophie (stricto sensu) correspondrait à un fonctionnement «à reste minimal» et à un transfert optimal de la matière assimilable disponible.

Au plan méthodologique, la tendance à la co-variation des deux indices et les discordances observées conduisent à rechercher un indice biologique résultant qui serait interprété à l'aide des deux indices Bo et $\mathrm{Bz}$ permettant de préciser le type biologique des lacs.

$\mathrm{Au}$ plan conceptuel, la mesure des flux d'énergiematière transférée au cours d'un cycle annuel se heurtant à de grandes difficultés (formes recyclées et différées) nous proposons de rechercher des descripteurs du mode de fonctionnement des systèmes par une analyse des conditions de transformation et de transfert de la matière assimilable aux organismes consommateurs.

\section{Travaux cités}

Alaoui-Mhamdi M. 1985 .- Dynamique des populations et évolution métabolique du phytoplancton d'un lac eutrophe (Lac d'Aydat, Puy-de-Dôme, France). Mém. $3^{\text {tme }}$ cycle, Univ. Clermont Il : $206 \mathrm{p}$.

Brundin L. 1949. - Chironomiden und andere Bodentiere der südschwedischen Urgebirgsseen. Inst. Freshwat. Res., Drottningholm, $30: 914 \mathrm{p}$.

Chessel D., Thioulouse J. \& Dolédec S. 1995 . ADE-4 program library, Multivariate and graphical display of environmental data, 6 vol., Univ., Lyon I, 520 p.

Dolédec S. \& Chessel D. 1994 .- Co-inertia analysis : an alternative method for studying species - environment relationships. Freshwat. Biol., 31 : 277-294.

Grote A. 1934 .- Über den Zusammenhang zwischen dem Sauerstoffhaushalt, den benthalfaunistischen Besiedelungsverhältnissen und der Typenzugehörigkeit der Seen. Int. Rev. ges. Hydrobiol. Hydrogr., 31 : 1-39.

Håkanson L. \& Jansson M. 1983 ._- Principles of Lake sedimentology. Springer-Verlag, Berlin, $316 \mathrm{p}$.

Hayes F.R. \& Philipps J.E. 1958 . - Lake water and sediment. IV Radio phosphorus equilibrium with mud, plants and bacteria under oxidized and reduced conditions. Limnol. Oceanogr. 3 : 459-475.

Hutchinson G.E. 1967 - - A Treatise on Limnology. II : Introduction to lake biology and limnoplankton. Wiley ed: New-York : $1115 \mathrm{p}$.

Johnson R.K. \& Wiederholm T. 1989.- Classification and ordination of profundal macroinvertebrate communities in nutrient poor, oligo-mesohumic lakes in relation to environmental data. Freshwat. Biol., 21: 275-386.

Lafont M., Juget J. \& Rofes G. 1991.- Un indice biologique lacustre basé sur l'examen des Oligochètes. Rev. Sci. Eau, 4 : 253-268.

Lundbeck J. 1926 . - Die Bodentierwelt norddeutscher Seen. Arch. Hydrobiol. Suppl. 7 : 1-473.

Martin C. 1994 .- Lacs de Franche-Comté (massif du Jura). Recherches sur le seston, plus particulièrement sur le trypton organique. Thèse d'état, Univ. Fr. Comté, Besançon, 205 p. 
Mouthon J. 1993. — Un indice biologique lacustre basé sur l'examen des peuplements de Mollusques. Bull. Fr. Pêche Pisc., 331 : 397-406.

Petersen R.C., Petersen L.M., Persson U., Kullberg A., Hargeby A. \& Paarlberg A.,1986.- Health aspects of humic compounds. Wat. Qual. Bull., $11: 44-62$

Prat N. 1978. - Benthos typology of Spanish reservoirs. Verh. Internat. Verein. Limnol., 20 : 1647-1651.

Rigler F.H. 1964 . - The phosphorus fractions and the turn-over time of inorganic phosphorus in different types of lakes. Limnol. Oceanogr., $9: 511-518$.

Ryding S.O. \& Rast W. 1994.- Le contrôle de l'eutrophisation des lacs et des réservoirs. Masson éd., Paris : 294 p.

Verneaux J., Remy F., Vidonne A. \& Guyard A. 1987.- Caractères généraux des sédiments de 10 lacs jurassiens. Rev. Sci. Eau, 6 : 107-128.
Verneaux J., Vidonne A., Remy F. \& Guyard A. 1991 .- Particules organiques et rapport $\mathrm{C} / \mathrm{N}$ des sédiments des lacs du Jura. Annls Limnol., 4 : 175-190.

Verneaux J., Verneaux V. \& Guyard A. 1993a.-Classification biologique des lacs jurassiens à l'aide d'une nouvelle méthode d'analyse des peuplements benthiques. I. Variété et densité de la faune. Annls Limnol. 29 : 59-77.

Verneaux V, Verneaux J. \& Guyard A. 1993b .- Classification biologique des lacs jurassiens à l'aide d'une nouvelle méthode d'analyse des peuplements benthiques. II. Nature de la faune. Annls Limnol. 29 : 383-393.

Weber C.A. 1907 . - Aufbau und Vegetation der Moore Norddeutschlands. Bot. Jahrb. $40: 19-32$.

Wetzel R.G. 1975 .- Limnology. Saunders ed., Philadelphia : 743 p. 